\title{
MISCELANEA
}

COMUNICACIÓN ORAL VII CONGRESO NACIONAL DE LA SOCIEDAD CIENTÍFICA ESPAÑOLA DE ENFERMERÍA - SCELE. Mayo de 2014. UNIVERSIDAD DE ALICANTE.

\section{PERCEPCIÓN DE ENFERMEDAD Y ADHERENCIA AL TRATAMIENTO EN PACIENTES CRÓNICOS POLIMEDICADOS DE ATENCIÓN PRIMARIA}

G. Canals Calbet, L. Rumí Belmonte, T. Diaz Espallardo, C. López Almela, MJ. Marco Navarro, L. Molina Albert.

Atenció Primària Parc Taulí. Corporació Sanitària Parc Taulí, Sabadell (Barcelona)

CORREO ELECTRÓNICO: Irumi@tauli.cat 


\section{PALABRAS CLAVE:} crónica.

Adherencia al tratamiento, percepción de enfermedad, polimedicación, enfermedad

\section{OBJETIVOS:}

- Conocer la percepción de enfermedad en los pacientes crónicos polimedicados desde Atención Primaria.

- Determinar si existe una mayor percepción de enfermedad en el grupo que recibe la intervención enfermera.

- Determinar si existe una mayor adhesión al tratamiento en el grupo que recibe la intervención enfermera.

\section{METODOLOGÍA:}

Hipótesis: Los pacientes crónicos (de 60 a 80 años) polimedicados ( $>5$ prescripciones indicadas desde Atención Primaria), que reciben una intervención enfermera para evaluar y promocionar la adhesión al tratamiento, presentan más percepción de enfermedad y por lo tanto mejor seguimiento al tratamiento que los pacientes que reciben la atención habitual en Atención Primaria.

Variables independientes:

- Intervención enfermera de valoración y promoción a la adhesión al tratamiento.

Variables dependientes:

- Percepción de enfermedad. (Resultado del cuestionario Medidas de Percepción en Enfermedades Crónicas IPQ-R, validado al español)

- Adhesión al tratamiento. (Resultado del cuestionario Escala de Adherencia Terapéutica, para pacientes con enfermedades crónicas, validado al español)

Variables para describir la muestra:

- Edad (años cumplidos) / Sexo /Patologías crónicas / Medicaciones crónicas / Nacionalidad (cultura).

Ensayo clínico controlado y aleatorio. La población son los pacientes crónicos de 60 a 80 años autónomos para las Actividades Básicas de la Vida Diaria (ABVD), polimedicados y con capacidades cognitivas conservadas, que se controlan en Atención Primaria Parc Taulí. El estudio está validado por el Comité Ético de Investigación Científica (CEIC) de la Corporació Sanitària Parc Taulí, previamente los pacientes que cumplen los criterios de inclusión reciben un consentimiento informado para entrar en el estudio. El análisis estadístico se realiza con el programa informático SPSS, la muestra se calcula para conseguir una potencia del $80 \%$ y para detectar diferencias en el contraste de la hipótesis nula mediante una prueba T-Student bilateral para dos muestras independientes.

\section{IMPLICACIONES PARA LA PRÁCTICA CLÍNICA:}

La enfermera de atención primaria, en el cuidado del enfermo crónico, se encuentra con frecuencia ante una falta de adherencia al tratamiento farmacólogo prescrito por el médico, así como a las prescripciones enfermeras de seguimiento, y/o, modificación de hábitos saludables (alimentación, actividad física). La OMS considera la falta de adherencia un tema prioritario de salud pública debido a sus múltiples consecuencias negativas: fracasos terapéuticos, mayores tasas de hospitalización, aumento de los costes sanitarios(Aguirrezabala 2011). Varios estudios relacionan la percepción de enfermedad del paciente como causa a la adherencia al tratamiento. La percepción de enfermedad es moldeada por factores socio culturales: como percibe, evalúa, se comporta, comunica y busca ayuda, es el resultado del proceso de socialización y experiencia adquirida (Di Silvestre 2013).

La falta de adhesión voluntaria al tratamiento comporta abandono del mismo, falta de compromiso y insatisfacción tanto del enfermo crónico como del profesional. Todo esto representa una carga innecesaria para el sistema de salud además de una perdida de tiempo y frustración para el personal sanitario (Di Silvestre 2013). 
Conocer las diferencias culturales en la percepción de la salud y la enfermedad, facilita la adherencia al tratamiento farmacólogo y la aceptación de las recomendaciones sobre cambios de estilo de vida.

En este proyecto de investigación queremos valorar la eficacia de un cambio de estrategia para la evaluación de la percepción de enfermedad y mejora de la adherencia al tratamiento prescrito tanto por la enfermera como por el médico.

\section{LIMITACIONES:}

Debido al diseño y selección de la muestra de forma aleatoria, podemos encontrarnos con el rechazo de los pacientes a participar en el estudio o en el seguimiento. La participación en el estudio no debe aumentar el número de visitas de enfermería. 